\title{
EL PARLAMENTO ITALIANO ENTRE REVISIONES Y TENTATIVAS DE REFORMA CONSTITUCIONAL
}

\author{
ALESSANDRO PACE
}


SUMARIO

1. «REVISIONES»Y REFORMAS» CONSTITUCIONALES. 2. LAS TENTATIVAS FALLIDAS DE «REFORMAS» CONSTITUCIONALES. 3. EL ARTÍCULO 138 DE LA CONSTITUCIÓN PREVÉ TODAVÍA LAS «REVISIONES» Y NO LAS «REFORMAS». 4. LA «REFORMA»BOSCHI. 


\title{
EL PARLAMENTO ITALIANO ENTRE REVISIONES Y TENTATIVAS DE REFORMA CONSTITUCIONAL
}

\author{
ALESSANDRO PACE ${ }^{1}$
}

\section{1. «REVISIONES»Y REFORMAS»CONSTITUCIONALES}

La Constitución de la República Italiana en el Título VI, bajo la rúbrica «Garantías constitucionales» (arts. 134-139), prevé dos secciones: la primera relativa a la Corte Constitucional (arts. 134-137); la segunda referente a la «revisión de la Constitución» y las «leyes constitucionales» (artículo 138).

Tanto la Corte Constitucional como las leyes de revisión constitucional constituyen la garantía de la Constitución: mientras la Corte Constitucional tiene la tarea de declarar la inconstitucionalidad de leyes y actos con fuerza de ley, las leyes de revisión constitucional, al modificar la Constitución, deben garantizar sus valores fundamentales. Y no deben subvertirlos ${ }^{2}$.

Significativa es, en este sentido, la aclaración —en las etapas finales de los trabajos de la Asamblea Constituyente- del Presidente Hble. Umberto Terracini dirigida al Hble. Costantino Mortati, autorizado constitucionalista, quien el 15 de enero

1 Catedrático emérito de Derecho constitucional de la Universidad «La Sapienza» de Roma. Doctor Honoris Causa por la Universidad de Oviedo. Facoltà di Giurisprudenza. Università di Roma La Sapienza. Piazzale Aldo Moro 5, 00185 Roma. Traducción del original italiano por Luis Jimena Quesada.

2 Para mayor comodidad del lector, transcribo a continuación el art. 138 de la Constitución italiana, relativo a las leyes constitucionales y de revisión constitucional:

«Las leyes de revisión de la Constitución y las demás leyes constitucionales serán aprobadas por cada una de las Cámaras en dos votaciones sucesivas, separadas por un intervalo no menor de tres meses, y por mayoría absoluta de los miembros de cada Cámara en la segunda votación.

Dichas leyes se someterán a referéndum popular cuando, dentro de los tres meses siguientes a su publicación, así lo solicite una quinta parte de los miembros de una Cámara, quinientos mil electores o cinco Consejos Regionales. La ley sometida a referéndum no se promulgará si no fuere aprobada con la mayoría de votos válidos.

No se celebrará el referéndum si la ley bubiese sido aprobada en la segunda votación efectuada por cada una de las Cámaras por una mayoría de dos tercios de sus respectivos componentes». 
de 1947 había propuesto considerar «la oportunidad de distinguir la revisión total de la Constitución de la revisión parcial». Pero el Presidente Terracini declinó la propuesta por ser «del parecer de que había que limitarse a la hipótesis de una revisión parcial» ${ }^{3}$.

En la misma reunión, el Hble. Luigi Einaudi —un muy autorizado economista y futuro primer Presidente de la República_- expresó el deseo de que las «Cámaras propongan una sola reforma a la vez y de manera clara para que los electores sean conscientes de lo que están llamados a votar» ${ }^{4}$.

Dicho esto, debe destacarse que, en italiano, el concepto de «revisión» alude a actividades precisas o, en todo caso, homogéneas. Por ejemplo, la revisión de un presupuesto, la revisión de una obra literaria, la revisión del motor de un automóvil ${ }^{5}$, mientras que el concepto de «reforma» alude —conceptualmente — a una modificación tal que «altera la estructura ordinaria de un determinado ámbito de la sociedad o de ésta en su conjunto, transformando sus reglas y leyes fundamentales» ${ }^{6}$.

En los primeros 35 años después de la entrada en vigor de la Constitución italiana, e incluso más tarde, las leyes de revisión constitucional tenían, todas ellas, un contenido preciso u homogéneo (así sucedía con las normas sobre control de constitucionalidad $^{7}$, las normas de desarrollo de la Constitución sobre la Corte Constitucio$\mathrm{nal}^{8}$, las modificaciones de los arts. 96, 134 y 135 en materia de procedimientos para exigencia de responsabilidad penal de miembros del Gobierno ${ }^{9}$, etc. ${ }^{10}$ ).

3 Vid. el acta de la sesión del 15 de enero de 1947 de la Sección II de la Subcomisión II de la Comisión Constitucional, en La Costituzione della Repubblica nei lavori preparatori della Assemblea costituente, Camera dei deputati, Roma, 1970, vol. VIII, p. 1876.

4 Ivi, p. 1878.

5 Según el autorizado Grande Dizionario della lingua italiana de S. BATTAGLIA, vol. XV, Utet, Torino, 1990, p. 969, se produce «revisión» en la hipótesis de verificación de la verdad (o de la conformidad) de unas cuentas económicas o de un presupuesto público o privado; se produce revisión asimismo en la hipótesis del reexamen en cuanto al fondo de un procedimiento judicial penal; se produce revisión en el examen textual o estilístico de una nueva edición o de la reimpresión de una obra literaria; se produce revisión en la operación de control y de mantenimiento de las máquinas o de los automóviles, etc.

6 Así en Wikipedia, voz Riforma (visitada el 17 de septiembre de 2017, a las 8.19 horas). En S. BATTAGLIA, Grande Dizionario, cit., vol. XVI, 1992, p. 279, el primer significado del vocablo es el siguiente: «modificación política, institucional o social de una ciudad, de un Estado, de un gobierno...».

7 Ley constitucional n. ${ }^{\circ} 1$ de 9 de febrero de 1948.

8 Ley constitucional n. ${ }^{\circ} 1$ de 11 de marzo de 1953.

9 Ley constitucional n. ${ }^{\circ} 1$ de 16 de enero de 1989.

${ }^{10}$ Ley constitucional n. ${ }^{\circ} 1$ de 23 de enero de 2001, de modificación de los arts. 56 y 57 de la Constitución; Ley constitucional n. ${ }^{\circ} 2$ de 31 de enero de 2001, mediante la que se aprueban disposiciones relativas a la elección directa el Presidente de las Regiones con estatuto especial; Ley constitucional n. ${ }^{\circ} 1$ de 20 de abril de 2012, mediante la que se introduce el principio del equilibrio presupuestario en la Corte Constitucional. 


\section{LAS TENTATIVAS FALLIDAS DE «REFORMAS» CONSTITUCIONALES}

No obstante, en octubre de 1983 la Cámara de los Diputados y el Senado, en la IX Legislatura, establecieron una Comisión parlamentaria bicameral presidida por el Senador Bozzi, quien en enero de 1985 presentó un proyecto para modificar unos 50 artículos de la Constitución, una «reforma» radical de la Constitución concerniente no solo al procedimiento legislativo, a las funciones de las Cámaras, a la disciplina de la confianza del Gobierno, etc., sino incluso a la Primera Parte de la Constitución relativa a los «derechos y deberes de los ciudadanos», que después de ello no fue nunca más cuestionada. El proyecto decayó con el final de la V Legislatura.

Ocho años después, en la XI Legislatura, las Cámaras aprobaron la Ley constitucional n. ${ }^{\circ} 1$ de 6 de agosto de 1993, mediante la que se establecía la Comisión parlamentaria para las reformas constitucionales —inicialmente presidida por el Hble. Ciriaco De Mita y luego por la Hble. Nilde Iotti- que debería haber elaborado «un proyecto orgánico de revisión constitucional relativo a la Parte II de la Constitución, con exclusión del Título VI ${ }^{11}$, así como los proyectos de ley sobre la elección de las Cámaras y de los Consejos de las Regiones con estatutos ordinarios». La Comisión trabajó en la reforma de la formación del Gobierno, de la moción de censura constructiva, de las nuevas reglas del presupuesto estatal, de la legislación de urgencia, de la relación entre el Estado y las Regiones, etc., pero decayó al final de la Legislatura sin dejar nada concluido.

La tercera tentativa de reforma constitucional tuvo lugar en la XIII Legislatura con la Ley constitucional n..$^{\circ} 1$ de 24 de enero de 1997 mediante la que se estableció la Comisión bicameral, presidida por el Hble. Massimo D’Alema. La ley preveía asimismo una reforma «orgánica» de unos 80 artículos con «quebrantamiento» (una especie de derogación de facto) del art. 138 de la Constitución, al someterla a ratificación popular. Al no tener efectos meramente temporales — característica esencial de las «derogaciones»—, la reforma de D’Alema preveía un «falsa» derogación ${ }^{12}$, porque habría derogado definitivamente, mediante esa reforma, las disposiciones vigentes de la Constitución. La reforma de D’Alema fue, por lo tanto, profundamente contradictoria e inconstitucional. Pues no solo violaba el art. 138 de la Constitución, sino también la libertad de voto (art. 48 de la Constitución) y la soberanía popular (art. 1 de la Constitución ${ }^{13}$ ), por cuanto los electores habrían tenido que decantarse en bloque

11 ... relativo a las «Garantías constitucionales».

12 Las reglas del ajedrez, con respecto a las cuales no difieren las reglas de la revisión constitucional — según las enseñanzas de Norberto Bobbio_- son típicamente reglas «constitutivas», en el sentido de que si, como en el juego del ajedrez, el caballo no puede moverse si no es de una cierta manera, por lo mismo las normas de revisión constitucional no pueden ser modificadas en el curso de la aprobación. En tal sentido, N. BOBBIO, I vincoli della democrazia, en Id., Il futuro della democrazia, Einaudi, Torino, 1984, p. 67.

${ }^{13}$ Vid. infra el $\S 4.1$.

(C) UNED. Revista de Derecho Politico

N.o 105, mayo-agosto 2019, págs 281-292 
por un solo Sí o un solo No para aprobar o rechazar la modificación de esos 80 artículos. Esta reforma fue igualmente abandonada debido a la confrontación surgida entre el Hble. Silvio Berlusconi, líder del «Polo della libertà», y el Hble. Massimo D’Alema, uno de los líderes del «Ulivo».

La cuarta tentativa de mega reforma fue llevada a cabo por el Gobierno Berlusconi con respeto al procedimiento del art. 138 de la Constitución (pero no con respeto sustancial, siendo una reforma y no una revisión). La reforma preveía la designación de un Primer Ministro «absoluto» ${ }^{14}$ que no solo habría podido designar o cesar a cada Ministro, sino que habría podido plantear autónomamente la cuestión de confianza $\mathrm{y}$ «perder la confianza» solo por «su propia» mayoría parlamentaria (¡sic!). La reforma también preveía un Senado federal, con elección de base regional y con poderes menores con respecto a la Cámara de los Diputados. La reforma Berlusconi fue aprobada por las Cámaras en 2005 (XIV Legislatura), pero fue rechazada en 2006 mediante referéndum por el 53,6 por ciento de los votos válidos de los electores.

Tras el transcurso de otros siete años, en 2013, el Primer Ministro Hble. Enrico Letta, al comienzo de la XVII Legislatura, propuso una «reforma» muy intricada que consistía, por una parte, en una ley constitucional de «revisión» que habría «derogado» (¡con efectos permanentes!) el procedimiento legislativo previsto por el art. 138 de la Constitución y, por otra parte, en una decena de leyes de «revisión» constitucional de varias materias (Parlamento, Presidente de la República, el Gobierno y las Regiones, etc.). Como ya sucedió con la reforma D’Alema (1997), el Hble. Berlusconi, por razones meramente políticas, le retiró la confianza al Gobierno Letta, que dejó decaer la ley de reforma.

Finalmente, en la misma XVII Legislatura Matteo Renzi, secretario político del «Partito Democratico» y Presidente del Consejo de Ministros aunque no elegido en el Parlamento, logró — con el cuestionable apoyo del entonces Presidente de la República Napolitano - aprobar, por una estrecha mayoría absoluta, la reforma Boschi — por el apellido de la Ministra de Reformas Constitucionales Hble. María Elena Boschi- de modificación de 53 artículos de la Constitución ${ }^{15}$.

Es menester recordar que, incluso antes de que comenzara el iter de la reforma Boschi, la Corte Constitucional había declarado mediante la Sentencia n. ${ }^{\circ} 1$ de 2014 la inconstitucionalidad de la Ley electoral n. 533 de 1993 (la conocida como «Porcellum» $)^{16}$ con la cual había sido elegida la XVII Legislatura. Solo los parlamentarios del «Movimento 5 Stelle» protestaron, pero sin éxito, por la grave violación infligida a la Constitución y a las normas parlamentarias, en la medida en que un Parlamento deslegitimado por la Corte pretendió llevar a cabo una reforma constitucional.

14 Así bautizado icásticamente por Leopoldo Elia, el muy autorizado constitucionalista y ex Presidente de la Corte Constitucional.

15 Vid. infra el $\$ 4$.

${ }^{16}$ El Hble. Roberto Calderoli de la «Lega Nord», que fue quien presentó el proyecto de ley, después promulgado como Ley n. ${ }^{\circ} 533$ de 20 diciembre de 1993, había calificado sin escrúpulos su ley como «una cerdada». 
La XVII Legislatura, en lugar de concluir entonces — como se imponía-, concluyó con las elecciones cuatro años después.

El referéndum constitucional sobre la reforma Boschi fue rechazado el 4 de diciembre de 2016 por el 65,5 por ciento de los votantes.

\section{EL ARTÍCULO 138 DE LA CONSTITUCIÓN PREVÉ TODAVÍA LAS «REVISIONES»Y NO LAS «REFORMAS»}

Antes de describir, siquiera brevemente, las claves de la reforma Boschi, resulta oportuna una puntualización.

Tras el fracaso de las seis reformas constitucionales mencionadas anteriormente, el art. 138 de la Constitución no ha perdido efectivamente el significado original, que atañe a «revisiones» precisas y en cualquier caso homogéneas.

Por el contrario, a nivel político-parlamentario, la valoración es diversa: hasta que una decisión parlamentaria certifique formalmente la invalidez de un procedimiento dado, incluso las mega reformas rechazadas y ni siquiera aprobadas son citadas y consideradas como «precedentes» parlamentarios.

\section{4. $\mathrm{LA} « \mathrm{REFORMA}$ » BOSCHI}

Procedo seguidamente a subrayar de modo muy sintético los puntos de la reforma Boschi merecedores de mayor crítica. A título de información complementaria me parece oportuno advertir que, en el curso de la campaña del referéndum contra la reforma Boschi, fui el Presidente de la Comisión por el «no».

4.1. La reforma Boschi, merced asimismo a la discutible Ley electoral $n .^{\circ} 52$ de 6 de mayo de 2015 — conocida como «Italicum» ${ }^{17}$, que sustituyó a la Ley «Porcellum»-, habría reforzado el papel del Primer Ministro, no tanto ampliando ulteriormente los poderes, ya notables en virtud de la Ley n. 400 de 23 de agosto de 1988, sino más bien disminuyendo los contrapoderes.

No obstante, en los procedimientos legislativos previstos por la reforma Boschi, que examinaré a continuación, el Gobierno habría disfrutado de una posición privilegiada.

4.2. El objetivo principal de la reforma Boschi radicaba en superar el bicameralismo parlamentario.

El Gobierno no habría quedado ya sometido a la confianza del Senado, sino solo a la confianza de la Cámara de los Diputados y mediante lo cual, gracias a la Ley

${ }^{17}$ La Corte Constitucional, mediante su Sentencia n. ${ }^{\circ} 35$ de 2017, ha declarado su inconstitucionalidad solo en parte. 
electoral «Italicum»y al hecho de que Renzi era el secretario del partido mayoritario, se habría instaurado un cargo de Primer Ministro «absoluto», que Leopoldo Elia había percibido ya en la tentativa de Berlusconi de 2006. Además, como veremos, como consecuencia de la reforma Boschi, las Regiones habrían perdido mucho poder con respecto al Estado central.

Mientras el número de miembros de la Cámara de los Diputados se habría mantenido en 630, los miembros del Senado se habrían reducido de 315 a 100, incluidos cinco nombrados por el Presidente de la República para siete años ${ }^{18}$.

Pese a que la Corte Constitucional hubiera reafirmado, mediante la Sentencia n. ${ }^{\circ} 1$ de 2014 - como principio supremo, y como tal intangible (Corte Constitucional, Sentencia n. ${ }^{\circ} 1146$ de 1986)_ que «la voluntad de los ciudadanos expresada a través del voto (...) constituye el principal instrumento de manifestación de la soberanía popular», la reforma Boschi habría eliminado el sufragio directo para la elección del Senado.

Los senadores habrían sido elegidos por los Consejos Regionales en número de 74 consejeros regionales y en número de 21 alcaldes. Los consejeros regionales y los alcaldes, sin embargo, habrían ejercido las funciones senatoriales solo a tiempo parcial. La duplicidad de funciones habría hecho prácticamente imposible el ejercicio de las importantes atribuciones, aunque limitadas, del Senado ${ }^{19}$.

Por lo demás, la disminución del número de senadores de 315 a 100 habría hecho irrelevante el voto de los senadores en el Parlamento actuando en sesión conjunta (730 miembros) para la elección del Presidente de la República, al requerirse dos tercios de los miembros del Parlamento en sesión conjunta, y para la elección de los miem-

${ }^{18}$ La motivación de los senadores vitalicios, prevista por el art. 59, párrafo 2, de la Constitución, y confirmada por la reforma Boschi, consistía en haber «ilustrado la Patria mediante altísimos méritos en el campo social, científico y literario», pese a que el Senado solamente representara a las «instituciones territoriales» (art. 55 de la Reforma Boschi).

19 Art. 55, párrafo 4: «El Senado de la República representa a las instituciones territoriales y ejerce funciones de conexión entre el Estado y los demás entes constitutivos de la República. Contribuye al ejercicio de la función legislativa en los casos y de acuerdo con las modalidades establecidas por la Constitución, así como al ejercicio de las funciones de conexión entre el Estado, los demás entes constitutivos de la República y la Unión Europea. Participa en las decisiones tendentes a la formación y ejecución de los actos normativos y de las políticas de la Unión Europea. Evalúa las políticas públicas y la actividad de las administraciones públicas y verifica el impacto de las políticas de la Unión Europea en los territorios. Participa en los nombramientos de competencia del Gobierno expresando su parecer en los casos previstos por la ley y contribuye en la supervisión de la aplicación de las leyes del Estado». [Resulta también de interés la lectura de la versión original italiana: «Il Senato della Repubblica rappresenta le istituzioni territoriali ed esercita funzioni di raccordo tra Stato e gli altri enti costitutivi della Repubblica. Concorre all'esercizio della funzione legislativa nei casi e secondo le modalità stabiliti dalla Costituzione, nonché all'esercizio delle funzioni di raccordo tra lo Stato, gli altri enti costitutivi della Repubblica e l'Unione europea. Partecipa alle decisioni dirette alla formazione e all'attuazione degli atti normativi e delle politiche dell'Unione europea. Valuta le politiche pubbliche e l'attività delle pubbliche amministrazioni e verifica l'impatto delle politiche dell'Unione europea sui territori. Concorre ad esprimere pareri sulle nomine di competenza del Governo nei casi previsti dalla legge e a verificare l'attuazione delle leggi dello Stato»]. Vid. asimismo los art. 70 y 71, párrafo 2, de la Reforma Boschi relativos a las competencias legislativas del Senado. 
bros no pertenecientes a la carrera judicial del Consejo Superior de la Magistratura, al exigirse dos tercios de los miembros del Parlamento en sesión conjunta y en votación secreta, o tres quintos de los miembros del Parlamento en sesión conjunta después de la tercera votación.

En lo que atañe a la elección de los magistrados constitucionales por el Parlamento en una sesión conjunta, ya no habrían sido elegidos los cinco por él, sino solo tres, mientras que los dos magistrados restantes habrían sido elegidos por los Consejos Regionales. Con la consecuencia de que estos dos magistrados habrían terminado defendiendo los intereses de las autonomías territoriales, poniendo de tal suerte en entredicho la imparcialidad de la Corte Constitucional.

4.3. Los procedimientos legislativos disciplinados por la reforma Boschi habrían sustituido a los tres actuales (el procedimiento ordinario, el de conversión de los decretos-ley y el constitucional) para pasar a ser nueve:

1) procedimiento de aprobación de las leyes constitucionales y de las leyes de revisión constitucional;

2) procedimiento bicameral paritario aplicable a los 16 ámbitos materiales enumerados en el art. 70, párrafo 1. Estas leyes, «cada una con objeto propio», podrían ser «suprimidas, modificadas o derogadas solo en forma expresa y mediante leyes aprobadas de conformidad con el presente párrafo»;

3) procedimiento tendencialmente monocameral, pero con la posible intervención del Senado en las demás materias (art. 70, párrafos 2 y 3);

4) procedimiento, favorable para el Gobierno, relativo a la denominada cláusula de «supremacía», tendencialmente monocameral, pero con la posible intervención del Senado dentro de los 10 días siguientes a la fecha de la transmisión por parte de la Cámara de los Diputados (art. 70, párrafo 4.);

5) procedimiento monocameral (no paritario) para las leyes de régimen presupuestario y financiero, pero con examen obligatorio del Senado y posibles propuestas de modificación, por parte del Senado, dentro de los 15 días a partir de la fecha de transmisión (art. 70, párrafo 5, y art. 81, párrafo 4);

6) procedimiento monocameral, favorable para el Gobierno, para los proyectos de ley con aprobación en «fecha determinada», no aplicable en cambio a las leyes electorales, a las leyes que autorizan la ratificación de los tratados, a las leyes de amnistía e indulto y a las leyes de presupuestos (art. 72, párrafo 7);

7) procedimiento de conversión de los decretos-ley con posible participación del Senado (art. 77, párrafos 2 y 3);

8) el procedimiento «especial» monocameral relativo a la aprobación de leyes electorales con posibilidad de control previo por parte de la Corte Constitucional (art. 73, párrafo 2);

9) procedimiento monocameral, activado por el Senado mediante votación por mayoría absoluta, requiriendo entonces a la Cámara de los Diputados para 
que proceda al examen de proyecto de ley que la Cámara deberá examinar y sobre el cual habrá debido «pronunciarse» dentro de los seis meses a partir de la fecha de aquella votación (art. 71).

La reforma Boschi, que se había propuesto el objetivo de simplificar el procedimiento de elaboración de las leyes considerado, no sin razón, demasiado farragoso en el sistema actual de bicameralismo perfecto, tuvo éxito al trazar como línea maestra el pasar de tres a nueve iter procedimentales distintos. Con el riesgo, según la mayoría de los académicos, de provocar conflictos de procedimiento no infrecuentes entre la Cámara y el Senado, que incluso podrían haber propiciado — dada la inadecuación del «acuerdo no disciplinado entre los presidentes de las dos Cámaras» («nuevo» art. 70, párrafo 6) — vicios de constitucionalidad, de naturaleza procedimental, sometidos a la competencia de la Corte Constitucional.

4.4. Por último, si bien temo que la doctrina se avenga a críticas menores, la reforma Boschi preveía una neta inversión de tendencia en las relaciones entre el Estado y las Regiones de régimen común con respecto a la Ley constitucional n. 3 de 2001 que, a la sazón, había aumentado los poderes de las Regiones. Por el contrario, las Regiones de régimen especial se habrían visto incluso «fortalecidas» con la reforma Boschi, ya que se previó el acuerdo necesario para las futuras modificaciones estatutarias.

Con respecto a las Regiones de régimen común, habría quedado suprimida la legislación concurrente, en virtud de la cual el Estado establece los principios y las Regiones dictan la normativa de detalle.

Por lo tanto, la reforma Boschi preveía devolver a la competencia exclusiva del Estado algunas materias atribuidas con demasiada generosidad a la competencia regional concurrente por la Ley constitucional n. 3 de 2001: los puertos y los aeropuertos civiles, las grandes redes de transporte y de navegación, la coordinación de las finanzas públicas y del sistema tributario y el ordenamiento de las comunicaciones.

Ahora bien, la reforma Boschi fue mucho más allá de esas modificaciones, adquiriendo dimensiones de una ... avalancha, arrastrando a la competencia estatal incluso aquellas materias que constituyen el corazón de cada ordenamiento autonómico, como las políticas sociales, la protección de la salud, la ordenación del territorio, el medio ambiente y el turismo.

El resultado eventualmente positivo del referéndum habría sido el siguiente: las materias atribuidas a la competencia legislativa exclusiva del Estado habrían alcanzado unas cincuenta, agrupadas en 21 letras de la a) a la z) del art. 117, párrafo 2, de la reforma Boschi.

A las Regiones se les habría atribuido, por el art. 117, párrafo 3, solo alrededor de 12 competencias legislativas, entre ellas la planificación del territorio regional; la programación y organización de los servicios sanitarios y sociales; la promoción y organización de la formación profesional en el ámbito regional; la promoción del 
derecho al estudio, incluido el nivel universitario; la promoción de los bienes ambientales, culturales y paisajísticos, etc. Materias que no revisten un carácter exclusivo, porque en tales materias habría sido atribuida al Estado la competencia legislativa sobre las disposiciones generales y comunes, de modo que la legislación concurrente que salió por la puerta... habría regresado por la ventana.

Ahora bien, el art. 116 de la reforma preveía que, a petición de las Regiones y siempre que mediaran «condiciones de equilibrio entre los ingresos y los gastos de sus presupuestos», se les atribuiría «ulteriores formas y condiciones particulares de autonomía», con la consecuencia de que las Regiones habrían quedado clasificadas en serie A y en serie B, por primera vez en Italia.

La lista de las competencias regionales exclusivas se completaba con una cláusula residual, en virtud de la cual a las Regiones — no al Estado- habría correspondido la potestad legislativa «en cualquier materia que no esté expresamente reservada a la competencia exclusiva del Estado».

Lo cual, ciertamente sin pretenderlo, habría comportado que se reservase a las Regiones materias de gran importancia, como la circulación en las carreteras, las obras públicas, la industria, la agricultura, la artesanía, la minería —incluida la extracción del petróleo-, las canteras, la caza y la pesca. Ello, sin embargo, como consecuencia de un olvido, no de una elección consciente a contracorriente.

Por consiguiente, cuando leí esta cláusula residual, me vino a la mente un viejo refrán italiano: «El diablo fabrica las ollas, pero no las tapas».

Title:

The Italian Parliament between revisions and attempts to reform

\section{Summary:}

1. Constitutional «Revisions» and «Reforms». 2. The failed attempts of constitutional «reforms». 3. Article 138 of the Constitution still provides for «Revisions» and not «Reforms». 4. The Boschi «Reform»

\section{Resumen:}

La hipótesis de partida del presente trabajo radica en que la Constitución italiana únicamente prevé «revisiones» constitucionales, homogéneas o incluso puntuales (apartado 1). No obstante, el autor critica que las fuerzas políticas han intentado aprobar hasta en seis ocasiones «reformas» constitucionales que, como tales, no son homogéneas. En concreto, cuatro de 
ellas no consiguieron ser aprobadas, mientras otras dos, aun siendo aprobadas por el Parlamento, acabaron siendo rechazadas tras sendos referéndums en 2006 y 2016 (apartado 2). Por lo tanto, el análisis revela que el significado original del concepto de «revisión» previsto en el artículo 138 de la Constitución italiana se ha mantenido firme (apartado 3). Finalmente, el autor somete a escrutinio la «reforma Boschi» (apartado 4), que fue abordada a iniciativa del líder del Partido Democrático, Matteo Renzi, siendo asimismo rechazada en sede popular. En efecto, dicha reforma planteaba serias perplejidades, tanto por la violación de la elección directa del Senado, como por la naturaleza contradictoria de la disciplina de los Senadores, por la diferenciación excesiva de los procedimientos legislativos y, por último, por la violación del principio constitucional de la garantía de las autonomías territoriales.

\begin{abstract}
:
This paper adopts as a starting hypothesis that the Italian Constitution only provides for constitutional, homogeneous or even occasional «revisions» (section 1). However, the author criticizes that political forces have intended to approve up to six times constitutional «reforms» that, as such, are not homogeneous. In particular, four of them failed to be approved, while two others, although approved by the Parliament, ended up being rejected after two referendums in 2006 and 2016 (section 2). Therefore, the analysis reveals that the original meaning of the concept of «revision» provided for in Article 138 of the Italian Constitution has remained firm (section 3). Finally, the author submits to scrutiny the so-called «Boschi reform» (section 4), which was addressed at the initiative of the leader of the Democratic Party, Matteo Renzi, and was finally rejected by popular referendum as well. Indeed, such reform posed serious perplexities, not only because of the violation of the direct election concerning the Senate, but also as a result of the contradictory nature of Senators statute, the excessive differentiation of legislative procedures and, finally, the violation of the constitutional principle recognizing the guarantee of self-government in favour of territorial entities.
\end{abstract}

Palabras clave:

Reforma Constitucional y Nociones Afines - Garantías Constitucionales Corte Constitucional - Procedimientos de Revisión - Referéndum - Papel del Parlamento

\title{
Key words:
}

Constitutional Amendment and Related Notions - Constitutional Guarantees - Constitutional Court - Revision Procedures - Referendum Role of Parliament 